\title{
Abnormal splicing of hepatocyte nuclear factor 1 alpha in maturity-onset diabetes of the young
}

\author{
M. P. Bulman 1 , L. W. Harries ${ }^{1 *}$, T. Hansen ${ }^{2}$, M. Shepherd ${ }^{1}$, W. F. Kelly ${ }^{3}$, A. T. Hattersley ${ }^{1}$, S. Ellard ${ }^{1}$ \\ ${ }^{1}$ Centre for Molecular Genetics, School of Postgraduate Medicine and Health Sciences, University of Exeter, Exeter, UK \\ 2 Steno Diabetes Center, Gentofte, Denmark \\ 3 The Diabetes Care Centre, Middlesbrough General Hospital, Middlesbrough, UK
}

\section{Abstract}

Aims/hypothesis. Mutations in the HNF-1 $\alpha$ gene result in maturity-onset diabetes of the young (MODY); an early-onset, dominantly inherited form of diabetes caused by pancreatic $\beta$-cell dysfunction. Splice site mutations represent approximately $10 \%$ of reported $H N F-1 \alpha$ mutations. No studies to date have investigated the effect of splice site mutations on mRNA processing because the tissues with abundant HNF$1 \alpha$ expression (liver, pancreas, kidney and gut) are not easily accessible for analysis. This study aimed to define the pathogenic mechanism in three novel splice site mutations by analysing illegitimate transcripts.

Methods. To assess the consequence of potential $H N F-1 \alpha$ splice site mutations we developed a nested reverse transcriptase PCR (RT-PCR) assay for the amplification of illegitimate $H N F-1 \alpha$ transcripts in Epstein Barr virus transformed lymphoblastoid cell lines.
Results. Sequencing the illegitimate $H N F-1 \alpha$ transcripts showed that the splice donor site mutation IVS8nt+1G $>$ A leads to complete skipping of exon 8 , the splice acceptor site mutation IVS4nt-2A $>$ G causes skipping of exon 5 with the recruitment of a cryptic splice acceptor site within intron 5 and the cryptic splice acceptor site mutation (IVS7nt-6G>A) resulted in the skipping of exon 7. All three changes are predicted to result in premature termination of the HNF$1 \alpha$ protein, providing further evidence for their role as pathogenic mutations.

Conclusion/interpretation. We conclude that the sequencing of illegitimate transcripts from lymphoblastoid cell lines is helpful in the assessment of intronic variation in $H N F-1 \alpha$ that could alter splicing. This analysis of the mRNA is required to define mutational mechanisms and confirm pathogenic status. [Diabetologia (2002) 45:1463-1467]

Keywords HNF1 $\alpha$, MODY, splicing, RT-PCR, illegitimate transcription.
Maturity-onset diabetes of the young (MODY) is a monogenic form of diabetes mellitus characterized by autosomal dominant inheritance, early age of onset

Received: 2 April 2002 / Revised: 5 June 2002

Published online: 11 September 2002

(C) Springer-Verlag 2002

*M.P. Bulman and L.W. Harries contributed equally to this paper. Corresponding author: Dr. A. T. Hattersley, Centre for Molecular Genetics, School of Postgraduate Medicine and Health Sciences, University of Exeter, Exeter, EX2 5AX, UK. E-mail: A.T.Hattersley@exeter.ac.uk

Abbreviations: HNF1 $\alpha$, Hepatocyte nuclear factor $1 \alpha$; EBV, Epstein Barr Virus. (usually $<25$ years) and pancreatic beta-cell dysfunction. There is genetic heterogeneity with mutations in six genes known to cause MODY; hepatocyte nuclear factor 4-alpha (HNF-4 $\alpha$; MODY1); glucokinase (MODY2), hepatocyte nuclear factor 1-alpha ( $H N F$ $1 \alpha$; MODY3), insulin promoter factor-1 (IPF-1; MODY4), hepatocyte nuclear factor 1-beta (HNF-1 $\beta$; MODY5) and Neuro D1 (MODY6). Heterozygous mutations in the Hepatocyte Nuclear Factor 1 alpha (HNF-1 $\alpha)$ gene are a common cause of maturity-onset diabetes of the young (MODY), accounting for approximately $63 \%$ of UK MODY families [1]. Since HNF-1 $\alpha$ mutations were first implicated as the cause of MODY3 in 1996, more than 120 different muta- 
tions have been described in the literature $[2,3] . \mathrm{Mu}-$ tations span the entire gene and most types of mutations have been described; e.g. insertion/deletion mutations, missense mutations, nonsense mutations and splice site mutations.

Splice site mutations disrupting the conserved splice donor (GT) and splice acceptor (AG) sites account for approximately $10 \%$ of HNF- $1 \alpha$ mutations described [3] and are therefore an important cause of MODY. No studies to date have investigated the effect of splice site mutations on mRNA transcription because the tissues with abundant HNF-1 $\alpha$ expression (liver, pancreas, kidney and gut) are not accessible for analysis. Illegitimate transcription (the presence of very low quantities of correctly spliced tissue specific mRNAs in non-expressing tissues) allows the examination of transcripts in more accessible tissues such as lymphocytes. A donor splice site mutation of the glucokinase gene has been studied through illegitimate transcription in lymphoblastoid cells [4] but this technique has not been applied to the analysis of HNF-1 $\alpha$ splicing mutations. In this study we describe the mutational mechanisms of three novel splice site mutations by analysis of mRNA from lymphoblastoid cell lines using a nested RT-PCR assay.

\section{Materials and methods}

Subjects. Subjects were chosen for sequencing of $H N F-1 \alpha$ if they had MODY according to the minimal criteria of at least one member of their family diagnosed with non-insulin-dependent diabetes mellitus under 25 years and an autosomal dominant pattern of inheritance with at least two generations affected. One family was previously reported as possible MODY but it was noted that there was considerable variation in the family in treatment, severity of beta-cell dysfunction and the presence of microvascular complications [5]. It is now accepted that this variation is a feature of many $H N F-1 \alpha$ mutations.

Direct sequencing. Direct sequencing was done using a previously described method [6], with alternative exon two primers to avoid allelic dropout [7]. Further studies were carried out on four pedigrees in which there were potential splice site abnormalities.

Establishment of transformed lines. The cell lines MY0376 (DUK102), MY0300 (DUK43), WT0652 (DUK129) and WT0654 (DUK179) were established from Epstein Barr virus (EBV) transformation of peripheral blood lymphocytes (PBLs) from three probands by the European Collection of Cell Cultures (Porton Down, Salisbury, UK).

Amplification of illegitimately transcribed RNA from transformed lymphoblastoid cells. Total RNA was extracted from approximately $1 \times 10^{6} \mathrm{EBV}$-transformed lymphoblastoid cells using the Perfect RNA Mini RNA kit (Eppendorf, Hamburg, Germany) according to manufacturer's instructions. Complementary DNA (cDNA) was synthesized from $4.5 \mu \mathrm{g}$ of the total RNA using the Thermoscript RT-PCR system (Life Technologies, Paisley, UK) according to the manufacturers instructions using $50^{\circ} \mathrm{C}$ as the incubation temperature. RT-PCR reactions were set up in triplicate in a two round nested PCR with three overlapping fragments covering the entire $H N F-1 \alpha$
cDNA. Four $\mu$ of each cDNA were used in each first step PCR reaction. For the second round, $1 \mu$ of undiluted first round product and $1 \mu \mathrm{l}$ of a 1:10 dilution were used. Conditions for both rounds of PCR were: $3 \mathrm{~min} 95^{\circ} \mathrm{C}$ initial denaturation, followed by 35 cycles of $95^{\circ} \mathrm{C} 1 \mathrm{~min}, 55^{\circ} \mathrm{C} 1 \mathrm{~min}, 72^{\circ} \mathrm{C} 2 \mathrm{~min}$ and a final $10 \mathrm{~min}$ extension at $72^{\circ} \mathrm{C}$. We used $2.5 \mathrm{U}$ of Taq polymerase (Abgene, Epsom, UK) per reaction.

Second round products were purified (Qiagen PCR Purification Kit, Quigen, Crawley, UK) and sequenced in both directions using Big Dye chemistry and an ABI 377 Sequencer (Applied Biosystems, Warrington, UK). An additional sequencing reaction was done using the M13 tail incorporated into the forward primer for the second round. Sequences were aligned with the published sequence using the Sequence Navigator software package (Applied Biosystems, Warrington, UK).

\section{Results}

Sequencing analysis of the coding region and splice sites of the $H N F-1 \alpha$ gene was done on United Kingdom probands with clinical features of MODY and patients with potential splice site mutations were selected for further study. Two families (DUK43 and 179 , see Fig. 1) had novel HNF-1 $\alpha$ mutations at conserved splice donor and acceptor sites (IVS8nt+1 $\mathrm{G}>\mathrm{A}$ and IVS4nt-2 $\mathrm{A}>\mathrm{G}$ ) [5] that cosegregated with diabetes in the families. Mutations at these conserved splice sites were predicted to result in exon skipping or intron retention, but examination of mutant mRNA transcripts was necessary in order to assess the precise consequence of these mutations. We have also found a potential novel cryptic splice site mutation (IVS7nt$6 \mathrm{G}>\mathrm{A})$ in probands from two unrelated families (DUK102 and 129). The variant, which creates a new consensus splice site, was also present in the proband's affected identical twin daughters in pedigree DUK102 but not found on testing 100 normal chromosomes. In the absence of mRNA analysis, the pathogenic status of this variant is uncertain.

In the absence of samples from tissues where there is legitimate transcription of $H N F-1 \alpha$, we attempted to use illegitimate transcription to examine mRNA expression in affected mutation carriers. Amplification of $H N F-1 \alpha$ mRNA from blood proved unsuccessful, so we therefore developed a two round nested RT-PCR approach in which the entire $H N F-1 \alpha$ mRNA was amplified from lymphoblastoid cell lines derived from MODY patients. Illegitimately expressed mRNA transcripts were amplified in three overlapping fragments from three probands in order to assess the pathological effect of each mutation. RT-PCR reactions were set up in triplicate from mRNA extracted from each lymphoblastoid cell line. Agarose gel electrophoresis of the RT-PCR products showed the presence of one product of the expected size for each reaction. In addition to the normal product, a single abnormal product was also observed in some reactions. All RT-PCR products were then analysed by sequencing (Table 1). 

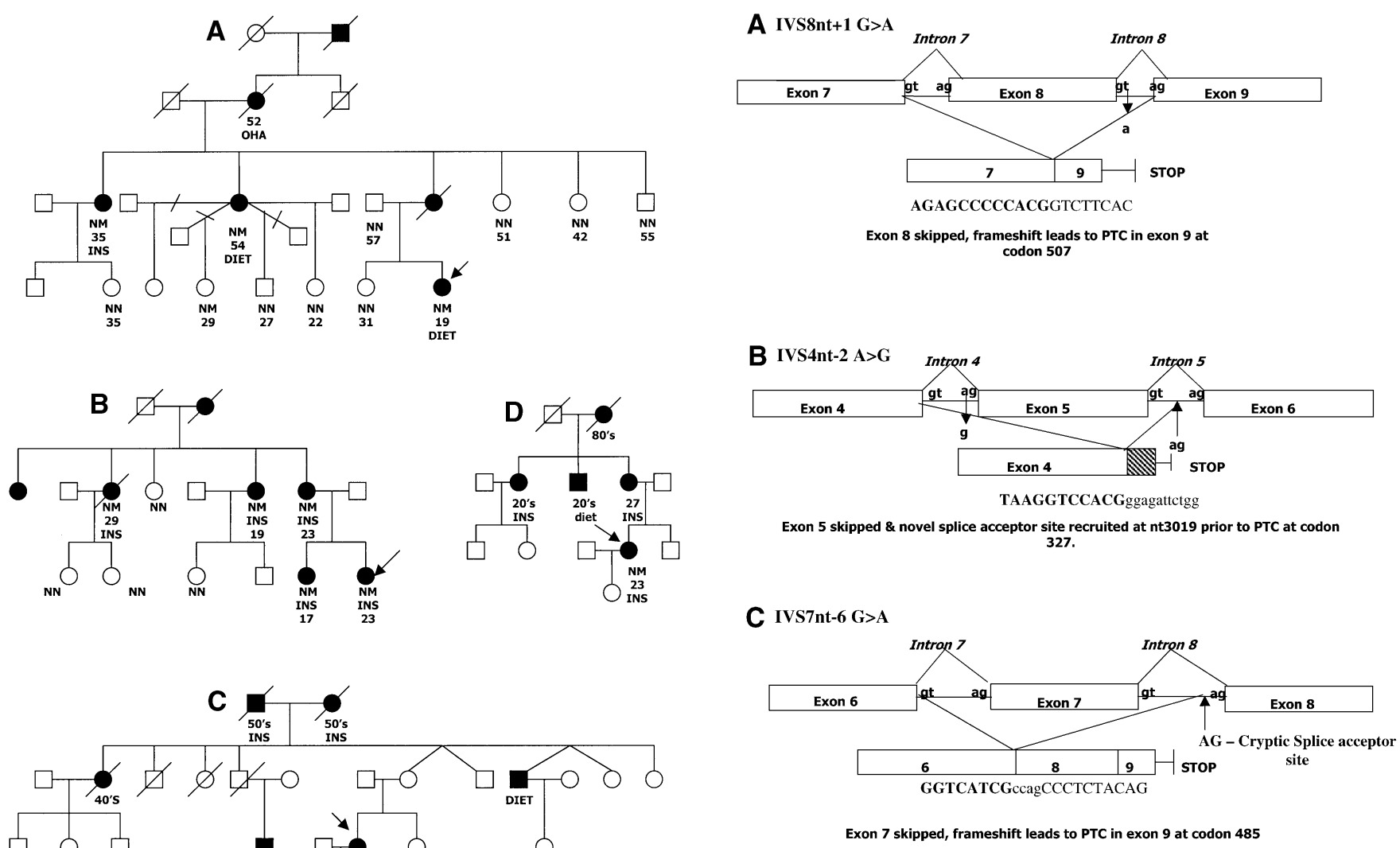

Fig. 2A-C. The predicted consequences of splice site and cryptic splice mutations in the $H N F-1 \alpha$ gene. A IVS8nt+ $1 \mathrm{G}>\mathrm{A} ; \mathbf{B}$ IVS4nt-2A>G; C IVS7nt-6G>A. This figure shows the intron:exon structure of the relevant region of the $H N F-1 \alpha$ gene in each case, together with the position of each mutation. The predicted structure of the transcript following splicing events is given, together with the true sequence of the junction region. The sequence of the $5^{\prime}$ and $3^{\prime}$ junctions are given in bold and normal capital letters respectively

Fig. 1A-D. Pedigrees for families in this study. A Family DUK43 (IVS8nt+1G>A) Family; B Family DUK179 (IVS4nt2A $>$ G); C Family DUK102 (IVS7nt-6G >A); D DUK129 (IVS7nt-6G $>$ A). Closed symbols represent affected subjects; open symbols represent unaffected subjects. Where tested, the mutation status of each individual is given $(N$, normal; $M$, mutation as given below). Age at diagnosis (where known) is given, together with treatment; diet, OHA (oral hypoglycaemic agents), INS (insulin). In unaffected members the age of testing is shown. All family histories are consistent with a diagnosis of MODY, with the age of diagnosis being before the age of 25 in most patients. RNA was isolated from cell lines derived from the proband, marked with an arrow in each case

Sequencing analysis of two of the three RT-PCR products for the intron 8 splice donor site mutation, $\mathrm{GT} \rightarrow \mathrm{AT}$ (IVS8nt+1G>A), showed only the normal allele. The third product showed a heterozygous sequence with complete skipping of exon 8 and the splicing of exons 7 and 9 in the mutant allele; the junction sequence is shown in Fig. 2A. The elimination of exon 8 led to a frameshift and a TGA premature termination in exon 9 at codon 507. This is the predicted pattern where skipping of the exon located immediately $5^{\prime}$ of the mutation occurs in the majority of +1 donor site mutations.

The intron 4 splice acceptor site mutation converting $A G$ to GG (IVS4nt-2A>G) led to skipping of exon

5 and the recruitment of a cryptic splice acceptor site within intron 5. This is predicted to result in truncation of the protein with the addition of 7 amino acids prior to a TAG stop site. The mutated sequence was again observed in the heterozygous state in only one of the three products, the other two products representing normal sequence only. This mutation might be expected to result in the skipping of the exon immediately $3^{\prime}$ to the mutation (exon 5) and the utilization of the next available AG site for exon 6. However, although exon 5 is indeed skipped, a cryptic AG splice acceptor site in intron 5-75 bp $5^{\prime}$ to the natural site was recruited resulting in inclusion of some intronic sequence and premature termination at codon 327; the junction sequence is shown in Fig. 2B.

The potential cryptic splice acceptor site mutation (IVS7nt-6G>A) showed a heterozygous sequence with splicing of exon 6 to exon 8 and the skipping of exon 7 on the mutant allele in one PCR product. In the other two products only the normal allele had been amplified. This mutation resulted in a frameshift with a TGA premature termination codon at 485 in 
Table 1. Primers used for nested RT-PCR of HNF-1 $\alpha$

\begin{tabular}{|c|c|c|}
\hline Primer set & Sense/antisense primer & \\
\hline \multirow[t]{8}{*}{ Round one } & Fragment $1-4 \mathrm{a}$ & \\
\hline & Ex1 FP & $5^{\prime}$ atggtttctaaactgagc $3^{\prime}$ \\
\hline & Ex4 RP & $5^{\prime}$ gcgggcagcgcaggtcc $3^{\prime}$ \\
\hline & Fragment 3-6a & \\
\hline & Ex3 FP & $5^{\prime}$ cagggcagggagggctga $3^{\prime}$ \\
\hline & Fragment 5-10a & \\
\hline & Ex5 FP & $5^{\prime}$ gacagcctgcgaccagtg $3^{\prime}$ \\
\hline & Ex10 RP & $5^{\prime}$ ttactgggaggaagaggc $3^{\prime}$ \\
\hline \multirow[t]{6}{*}{ Round two } & Fragment $1-4 b$ & \\
\hline & Ex1 FS & $5^{\prime}$ tgtaaaacgacggccagtctcctggcggccetgctc $3^{\prime}$ \\
\hline & Ex6 RS & $5^{\prime}$ tgttggtgaacgtaggac $3^{\prime}$ \\
\hline & Fragment $5-10 b$ & \\
\hline & Ex5 FS & $5^{\prime}$ tgtaaaacgacggccagttaccctcaagcagcggcg 3' \\
\hline & Ex10 RS & $5^{\prime}$ ctgggtggagatgaaggt $3^{\prime}$ \\
\hline
\end{tabular}

M13 5' tails are shown in underlined italics in the forward round 2 primers

exon 9 , as shown by the junction sequence given in Fig. 2C.

\section{Discussion}

This report describes the first $H N F-1 \alpha$ splice site mutations to be studied at the mRNA level from MODY patients. All three mutations result in a transcript showing abnormal splicing and result in a premature termination codon. These mutations are very likely to be pathogenic as the resulting truncated protein will probably result in loss of function.

It is interesting that for each of the three mutations the mutant allele is frequently not amplified. The reason for this is uncertain. It is not a consequence of the illegitimate transcription technique used to study the RNA, since when we examined RNA from a normal individual heterozygous for a polymorphism in exon 7 of the $H N F-1 \alpha$ gene $(1375 \mathrm{C}>\mathrm{T})$ both alleles were detected in $35 / 40(87.5 \%)$ replicate RT-PCR reactions. One explanation is that there is less RNA available for amplification from the mutant allele compared to the normal allele. In vitro studies after the introduction of some $H N F-1 \alpha$ mutations into cell lines have resulted in reduced protein levels 8]. For the mutations we describe, a possible explanation is that mutant transcripts containing premature termination codons are detected and degraded by nonsense mediated decay (NMD), a ubiquitous process in eukaryotic cells [9]. NMD has been reported as a result of splice site mutations [10].
Further study of the splice site mutations and other changes that result in a premature termination codon are warranted to determine whether NMD is an important mechanism associated with $H N F-1 \alpha$ mutations.

We have shown the utility of using illegitimate transcription of HNF-1 $\alpha$ in transformed lymphocytes for determining the consequences of splice site mutations and investigating the pathogenic status of intronic mutations occurring outside the conserved splice donor and acceptor sites. All three mutations show alternative splicing that is predicted in addition to the exclusion of exons to also result in premature termination of the protein, thus providing further evidence for their role as pathogenic mutations. These findings highlight the importance of investigating intronic changes and their consequences at the mRNA level to ascertain mutational mechanisms and confirm pathogenic status.

Acknowledgements. We are grateful to Diabetes UK, The European Union (Contract number QLG-CT-1999-00546 [GIFT]), the Royal Devon and Exeter NHS Healthcare trust, The European Collection of Cell Cultures (ECACC) and the University of Exeter for financial support.

\section{References}

1. Frayling TM, Evans JC, Bulman MP et al. (2001) Beta cell genes and diabetes - molecular and clinical characterisation of mutations in transcription factors. Diabetes 50 [Suppl 1] 
2. Fajans SS, Bell GI, Polonsky KS (2001) Molecular mechanisms and clinical pathophysiology of maturity-onset diabetes of the young. N Engl J Med 345: 971-980

3. Ellard S (2000) HNF-1alpha mutations in MODY. Hum Mutat 16: 377-385

4. Sun F, Knebelmann B, Pueyo ME et al. (1993) Deletion of the donor splice site of intron 4 in the glucokinase gene causes maturity-onset diabetes of the young. J Clin Invest 92: $1174-1180$

5. Bodansky JH, Kelly WF (1987) Familial diabetes mellitus with variable $\mathrm{B}$ cell reserve: analysis of a pedigree. Diabetologia 30: 638-640

6. Frayling TM, Bulman MP, Appleton M, Bain SC, Hattersley AT, Ellard S (1997) A rapid screening method for hepatocyte nuclear factor 1 alpha; prevalence in maturity-onset diabetes of the young and late-onset non-insulin dependent diabetes. Hum Genet 101: 351-354
7. Ellard S, Bulman MB, Frayling TM et al. (1999) Allelic drop-out in exon 2 of the hepatocyte nuclear factor- 1 alpha gene hinders the identification of mutations in three families with maturity-onset diabetes of the young. Diabetes 48 : 921-923

8. Vaxillaire M, Abderrahmani A, Boutin P et al. (1999) Anatomy of a homeoprotein revealed by the analysis of human MODY3 mutations. J Biol Chem 274: 35639-35646

9. Frischmeyer PA, Dietz HC (1999) Nonsense-mediated mRNA decay in health and disease. Hum Mol Genet 8: 1893-1900

10. Bouma P, Cabral WA, Cole WG, Marini JC (2001) COL5A1 exon 14 splice acceptor mutation causes a functional null allele, haploinsufficiency of alpha $1(\mathrm{~V})$ and abnormal heterotypic interstitial fibrils in Ehlers-Danlos syndrome II. J Biol Chem 276: 13356-13364 